\title{
MEASURABILITY PROPERTIES OF SPECTRA
}

\author{
S. LEVI $^{1}$ AND Z. SLODKOWSKI
}

\begin{abstract}
We study Borel measurability of the spectrum in topological algebras. We give some equivalences of the various properties, show that the spectrum in a Banach algebra is continuous on a dense $G_{\delta}$, and prove that in a Polish algebra the set of invertible elements is an $F_{\sigma \delta}$ and the inverse mapping is a Borel function of the second class.
\end{abstract}

This article has its origin in the papers [7] and [5]. We study Borel measurability of the spectrum and related sets and mappings in various classes of algebras.

The best-known example is the case of Banach algebras: the spectrum is then an upper semicontinuous mapping, the set of invertible elements open, and the inverse mapping continuous. In the first part of the paper we establish relations between various measurability properties and study the set of points of measurability of the spectrum considered as a set-valued function. (This part of the paper-in particular Lemma 6 through Corollary 9-can be read independently.) We prove, among other things, the following fact.

TheOREM (CF. THEOREM 7). If $X$ is a Banach algebra, then the spectrum $x \rightarrow \sigma(x)$ : $X \rightarrow 2^{\mathrm{C}}$ is continuous on a dense $G_{\delta}$ in $X$.

The second part of the paper uses topological methods to deduce measurability results: we prove that in a Polish algebra the set of invertible elements is an $F_{\sigma \delta}$ and the inverse mapping is a Borel measurable function of the second class.

We prove in particular (Theorem 13), without any assumption of separability or local convexity, that the spectrum of a continuous linear operator acting on a complete metrizable vector space is always a $G_{\delta \sigma}$ set. This answers a question posed by A. L. Shields [6].

Terminology. Throughout the paper $X$ will denote a complex algebra with identity $e$ (commutativity is not assumed), which is also a topological vector space.

The continuity properties of multiplication will be specified case by case.

An $F$-space is a complete metric vector space.

A Polish space is a complete separable metric space.

A topological space $Z$ is said to be a Baire space if every nonempty open subset of $Z$ is of second category (i.e. cannot be represented as the union of a countable family of nowhere dense subsets of $Z$ ).

Received by the editors January 18, 1985 and, in revised form, August 21, 1985.

1980 Mathematics Subject Classification. Primary 46H05; Secondary 54H05.

${ }^{1}$ The first author is a member of G.N.A.F.A. (C.N.R.). 
Let $Y$ and $Z$ be topological spaces and $B: Y \rightarrow 2^{Z}$ a set-valued mapping. The graph of $B$ is

$$
\operatorname{Gr} B=\{(y, z) \in Y \times Z: z \in B(y)\}
$$

and if $V \subset Z, B^{-1}(V)=\{y \in Y: B(y) \cap V \neq \varnothing\}$.

$B$ is lower semicontinuous (lsc) at $y \in Y$ if for every $V$ open in $Z$ such that $V \cap B(y) \neq \varnothing, y \in$ int $B^{-1}(V)$.

$B$ is upper semicontinuous (usc) at $y \in Y$ if for every $V$ open in $Z$ such that $B(y) \subset V$, there exists a neighborhood $U$ of $y$ with $B(t) \subset V$ for each $t$ in $U$.

$B$ is continuous if it is both lsc and usc.

The maps $\Pi_{Y}$ and $\Pi_{Z}$ on the product $Y \times Z$ denote the natural projections.

The complement of a set $A$ is denoted by $C A$.

The inverse mapping in the algebra $X$ is denoted by $x \rightarrow x^{-1}$.

Let us recall that $X$ is a complex algebra and a topological vector space, $W$ is the set of invertible elements of $X$, and $\sigma: X \rightarrow 2^{\mathrm{C}}$ is the spectrum mapping: $\sigma(x)=\{\lambda$ $\in \mathbf{C}: x-\lambda e \notin W\}$.

THEOREM 1. The following conditions are equivalent:

(i) $W$ is open.

(ii) $\mathrm{Gr} \sigma$ is closed in $X \times \mathbf{C}$.

(iii) $\sigma$ is upper semicontinuous.

Proof. Define $\theta: X \times \mathbf{C} \rightarrow X$ by $\theta(x, \lambda)=x-\lambda e ; \theta$ is continuous, and the equivalence of (i) and (ii) follows from the equalities

$$
\theta^{-1}(W)=C \mathrm{Gr} \sigma \quad \text { and } W=\Pi_{X}\left[\theta^{-1}(W) \cap(X \times\{0\})\right] .
$$

If $\sigma$ is usc, then $W$ is open because $C W=\sigma^{-1}(\{0\})$.

Suppose, conversely, that $W$ is open; then $V=\{x \in X: \sigma(x) \subset \mathbf{C} \backslash\{1\}\}=W+$ $e$ is an open neighborhood of 0 in $X$.

Since the map $(x, \lambda) \rightarrow \lambda x(x \in X, \lambda \in \mathbf{C})$ is continuous, there exist $\varepsilon>0$ and a neighborhood $D$ of 0 in $X$ such that $\bar{B}(0, \varepsilon) \times D \subset V$, where $\bar{B}(0, \varepsilon)=\{t \in \mathbf{C}$ : $|t| \leqslant \varepsilon\}$. Fix $x$ in $D$ : then for every $|t| \leqslant \varepsilon, t \neq 0,1 \notin \sigma(t x)$; that is, $1 / t \notin \sigma(x)$ and $\sigma(x) \subset B(0,1 / \varepsilon)$. Thus $\sigma$ is uniformly bounded on a neighborhood of zero.

Suppose $\sigma$ is not usc at a point $x_{0} \in X$ : then there exist an open neighborhood $G$ of $\sigma\left(x_{0}\right)$ in $\mathbf{C}$ and nets $x_{j} \rightarrow x_{0}$ and $\lambda_{j} \in \sigma\left(x_{j}\right) \backslash G$. Pick $n$ in $\mathbf{N}$ such that $x_{0} \in n D$ and determine $j_{0}$ such that $\forall j \geqslant j_{0} x_{j} \in n D$; it follows that $\left|\lambda_{j}\right| \leqslant M$ for $j \geqslant j_{0}$ and some $M>0$. Extract a subnet $\left\{\lambda_{j_{k}}\right\}$ converging to $\lambda$. By the equivalence of (i) and (ii), Gr $\sigma$ is closed. Therefore $\lambda \in \sigma\left(x_{0}\right) \subset G$, a contradiction.

COROLLARY 2. If any of the conditions of Theorem 1 is verified, $\sigma(x)$ is compact for every $x$ in $X$, and $\sigma$ maps compact sets into compact sets.

Proof. Since $\sigma$ has a closed graph, it carries compact sets into closed sets. Moreover the image of a compact set is bounded since $\sigma$ is uniformly bounded on a balanced neighborhood of zero in $X$. 
THEOREM 3. The following conditions are equivalent:

(i) $W$ is $a G_{\delta}$.

(ii) Gro is an $F_{\sigma}$.

(iii) $\sigma^{-1}(F)$ is an $F_{\sigma}$ in $X$ for every closed subset $F$ of $\mathbf{C}$.

If $X$ is an $F$-space with continuous multiplication, the next condition is also equivalent to the above three:

(iv) the mapping $x \rightarrow x^{-1}$ is continuous.

The proof of Theorem 3 is similar to the proof of Theorem 5 .

The equivalence of (iv) and (i) when $X$ is an $F$-space is due to Banach [3].

REMARK 4. Let $\mathbf{C}(t)$ be the quotient field of the algebra of complex polynomials in $t$. It is possible to define a metrizable locally convex topology on $\mathbf{C}(t)$ such that multiplication is jointly continuous and the set of invertible elements is open but such that the mapping $x \rightarrow x^{-1}$ is not continuous. For the details see [2, pp. 277, 279].

This shows that the completeness assumption in Theorem 3 is essential.

As for Borel sets of the second class, we have

THEOREM 5. Suppose that the topology of $X$ is completely metrizable. Then the following conditions are equivalent:

(i) $W$ is an $F_{\sigma \delta}$.

(ii) Gro is $a G_{\delta \sigma}$

(iii) $\sigma^{-1}(F)$ is $a G_{\delta \sigma}$ for every closed subset $F$ of the plane.

Proof. Assume (ii). Thus Gro $=\cup_{m} G_{m}$, where each $G_{m}$ is a $G_{\delta}$ in $X \times \mathbf{C}$. Let $\left(K_{n}\right)$ be a sequence of compact subsets of $\mathbf{C}$ such that $\mathbf{C}=\bigcup_{n} K_{n}$. Then

$$
\sigma^{-1}(F)=\Pi_{X}[(X \times F) \cap \mathrm{Gr} \sigma]=\bigcup_{n} \bigcup_{m} \Pi_{X}\left[\left(X \times\left(F \cap K_{n}\right)\right) \cap G_{m}\right] .
$$

Note that $\left(X \times\left(F \cap K_{n}\right)\right) \cap G_{m}$ is a $G_{\delta}$ in $X \times \mathbf{C}$ and is therefore completely metrizable. The mapping $\Pi_{X \mid X \times K_{n}}$ is closed and continuous.

We use now the following result of Vainstein [8, p. 320, Theorem 6]: if $f$ is a continuous closed mapping of a complete metric space $Z$ onto a metrizable space $W$, then $W$ is completely metrizable (and so an absolute $G_{\delta}$ ). It follows that $\Pi_{X}\left[\left(X \times\left(F \cap K_{n}\right)\right) \cap G_{m}\right]$ is a $G_{\delta}$ in $X$.

The formula for $\sigma^{-1}(F)$ now concludes the proof.

We will show below that if $X$ is a Polish algebra with continuous multiplication, the above conditions are fulfilled.

LEMMA 6. Suppose that $X$ is a Baire space and $W$ is $a G_{\delta}$. Then $\sigma$ is lower semicontinuous on a dense $G_{\delta}$ in $X$.

Proof. By Theorem 3, $\sigma^{-1}(F)$ is an $F_{\sigma}$ in $X$ for each closed subset $F$ of the plane. Since open subsets of $\mathbf{C}$ are $F_{\sigma}$ 's, their preimages under $\sigma$ are $F_{\sigma}$ 's too.

The mapping $\sigma$ is not lsc at $x_{0} \in X$ if there exists an open $V \subset \mathbf{C}$ such that $V \cap \sigma\left(x_{0}\right) \neq \varnothing$ and $x_{0} \notin$ int $\sigma^{-1}(V)$.

Let $K=\{x \in X: \sigma$ is not lsc at $x\}$. Then

$$
K=\bigcup\left\{\left[\sigma^{-1}(V) \backslash \text { int } \sigma^{-1}(V)\right]: V \text { is open in } \mathbf{C}\right\} \text {. }
$$


Each set $\sigma^{-1}(V) \backslash$ int $\sigma^{-1}(V)$ is an $F_{\sigma}$ of the first category, and since $\mathbf{C}$ has a countable base the same conclusion holds for $K$. The lemma now follows because $X$ is a Baire space.

As a consequence of Lemma 6 and Theorem 1, we have

THEOREM 7. Suppose $X$ is a Baire space and $W$ is open. Then $\sigma$ is continuous on $a$ dense $G_{\delta}$.

REMARK 8. (i) By Corollary 2, $\sigma(x)$ is compact for each $x$ in $X$.

Thus $\sigma$ is lsc and usc if and only if it is continuous, as a point-map, with respect to the Hausdorff metric in the plane.

(ii) The hypotheses of Theorem 7 are verified by Banach algebras. We have, as a consequence, that the spectral radius is continuous on a dense $G_{\delta}$. For examples of spectral discontinuities in Banach algebras see [1].

COROLlary 9. Suppose $X$ is a Banach algebra. If the set $M$ of points of continuity of the spectrum is closed under addition, it coincides with the whole algebra.

Proof. Since the origin belongs to $M$, the assumption implies that $M$ is a vector subspace. By Theorem 7, $M$ is topologically complete and, hence, by a result of Klee [4], it is complete. Therefore $M=X$.

Let $Z$ be a Polish space and $X_{0}$ a subset of $Z$.

Definition 10 [7]. A family $\left(E_{n}^{j}: j, n \in \mathbf{N}\right)$ of subsets of $X_{0}$ is a regular system for $X_{0}$ if the following conditions hold:

(i) each $E_{n}^{j}$ is relatively closed in $X_{0}$,

(ii) $E_{n}^{j} \subset E_{n}^{j+1} \forall n, j \in \mathbf{N}$,

(iii) $\cup_{j} E_{n}^{j}=X_{0} \forall n \in \mathbf{N}$,

(iv) for each $\sigma=\left(\sigma_{n}\right) \in \mathbf{N}^{\mathbf{N}}, E(\sigma)=\bigcap_{n} E_{n}^{\sigma_{n}}$ is closed in $Z$.

In [6] it is proved that a subset of $Z$ which admits a regular system is a Borel subset of $Z$.

Using the results of [5] we can prove

THEOREM 11. A subset of $Z$ which admits a regular system is $F_{\sigma \delta}$ in $Z$.

For the proof we need

LEMMA 12. Let $Y$ be a metric space and suppose that $B: \mathbf{N}^{\mathbf{N}} \rightarrow 2^{Y}$ is a set-valued mapping satisfying the following conditions:

(i) $\forall \sigma \in \mathbf{N}^{\mathbf{N}}, \boldsymbol{B}(\boldsymbol{\sigma})$ is closed in $Y$,

(ii) if $\sigma \leqslant \tau$ in $\mathbf{N}^{\mathbf{N}}$ (that is $\sigma_{n} \leqslant \tau_{n} \forall n \in \mathbf{N}$ ), $B(\sigma) \subseteq B(\tau)$,

(iii) if $\sigma^{1} \geqslant \sigma^{2} \geqslant \cdots$ and $\sigma^{k} \rightarrow \sigma$ in $\mathbf{N}^{\mathbf{N}}, B(\sigma)=\bigcap_{k} B\left(\sigma^{k}\right)$, then the graph of $B$ is closed in $\mathbf{N}^{\mathbf{N}} \times Y$.

Proof. Let $\sigma^{k} \rightarrow \sigma$ and $y_{k} \in B\left(\sigma^{k}\right)$ with $y_{k} \rightarrow y$ in $Y$. We must show that $y \in B(\sigma)$. 
Define $\tilde{\boldsymbol{\sigma}}^{k} \in \mathbf{N}^{\mathbf{N}}$ for every $k=1,2, \ldots$ by putting

$$
\tilde{\sigma}_{n}^{k}=\sup \left\{\sigma_{n}^{m}: m \geqslant k\right\} \text {. }
$$

Clearly $\tilde{\boldsymbol{\sigma}}^{k} \geqslant \tilde{\boldsymbol{\sigma}}^{k+1}$ for every $k$, and $\lim \tilde{\boldsymbol{\sigma}}^{k}=\boldsymbol{\sigma}$. Therefore $B\left(\tilde{\boldsymbol{\sigma}}^{k+1}\right) \subset B\left(\tilde{\boldsymbol{\sigma}}^{k}\right)$ for every $k$ and

$$
B(\sigma)=\bigcap_{k \geqslant 1} B\left(\tilde{\sigma}^{k}\right)
$$

On the other hand, $\sigma^{m} \leqslant \tilde{\sigma}^{m} \leqslant \tilde{\sigma}^{k}$ whenever $m \geqslant k$, and so $B\left(\sigma^{m}\right) \subset B\left(\tilde{\sigma}^{k}\right)$ for $m \geqslant k$. This implies that for each $k$ the closed set $B\left(\tilde{\sigma}^{k}\right)$ contains the sequence $\left(y_{m}\right)_{m \geqslant k}$ together with its limit $y$. Therefore $y \in B(\sigma)$.

Proof of TheOREM 11. Let $E: \mathbf{N}^{\mathbf{N}} \rightarrow 2^{Z}$ be the set-valued mapping $E(\sigma)=$ $\bigcap_{n} E_{n}^{\sigma_{n}}$, where $\sigma=\left(\sigma_{n}\right)_{n} \in \mathbf{N}^{\mathbf{N}}$. Gr $E$ is closed in $\mathbf{N}^{\mathbf{N}} \times Z$ as a consequence of Lemma 12. Thus Gr $E$ is a Polish space. Let us put $\pi=\pi_{Z \mid \mathrm{Gr} E} \cdot \pi$ is continuous and $\pi(\mathrm{Gr} E)=X_{0}$, by property (iii) of regular systems and the equality $\cup_{\sigma} \cap_{n} E_{n}^{\sigma_{n}}=$ $\bigcap_{n} \cup_{\sigma} E_{n}^{\sigma_{n}}$.

Let us show that $\pi$ maps open subsets of $\mathrm{Gr} E$ onto $F_{\sigma}$ sets.

Let $M=\left\{\sigma \in \mathbf{N}^{\mathbf{N}}: \sigma_{1}=c_{1}, \ldots, \sigma_{k}=c_{k}\right\}$ be a basic open set in $\mathbf{N}^{\mathbf{N}}$ and let $V$ be open in $Z$. It is enough to show that $\pi[(M \times V) \cap \mathrm{Gr} E]$ is $F_{\sigma}$. But

$$
(M \times V) \cap \operatorname{Gr} E=\left\{(\sigma, x): x \in E(\sigma) \cap V ; \sigma_{1}=c_{1}, \ldots, \sigma_{k}=c_{k}\right\}
$$

and

$$
\begin{aligned}
\pi[(M \times V) \cap G r E] & =\bigcup_{\sigma \in M}(E(\sigma) \cap V)=\bigcup_{\sigma \in M}\left(\bigcap_{n} E_{n}^{\sigma_{n}} \cap V\right) \\
& =V \cap\left(\bigcup_{\sigma \in M} \bigcap_{n} E_{n}^{\sigma_{n}}\right)=V \cap\left(\bigcap_{n} \bigcup_{\sigma \in M} E_{n}^{\sigma_{n}}\right) \\
& =V \cap E_{1}^{c_{1}} \cap E_{2}^{c_{2}} \cap \cdots \cap E_{k}^{c_{k}}
\end{aligned}
$$

by property (ii) of regular systems.

This shows that $\pi[(M \times V) \cap \mathrm{Gr} E]$ is $F_{\sigma}$ in $X_{0}$, since each $E_{k}^{c_{k}}$ is closed in $X_{0}$. By Theorem 2 of [5] we can conclude that $X_{0}$ is $F_{\sigma \delta}$ in $X$.

As an immediate corollary we have

THEOREM 13. Let $T$ be a continuous linear operator on an F-space. Then the spectrum of $T$ is $G_{\delta \sigma}$ in the plane.

Proof. As shown in [7], the resolvent of $T$ admits a regular system. Thus, by the preceding theorem, it is $F_{\sigma \delta}$ in $\mathbf{C}$.

Theorem 13 sharpens the conclusions of [7], where $\sigma(T)$ was shown to be a Borel subset of the plane and proved a $G_{\delta \sigma}$ only in the separable or locally convex case.

The preceding result naturally leads to the following open question: is every $G_{\delta \sigma}$ of the plane the spectrum of a continuous linear operator on an $F$-space?

THeOREM 14. Let $X$ be a Polish algebra with continuous multiplication. Then $W$ is $F_{\sigma \delta}$ in $X$, and the mapping $x \rightarrow x^{-1}$ is a Borel measurable function of the second class. 
Proof. Let $d$ be a complete invariant metric on $X$, and for each $k, n$ in $N$ let

$$
\begin{aligned}
& E_{n}^{k}=\left\{a \in X: \forall x \in X, d(0, x)>1 / 2^{n} \Rightarrow d(0, a x) \geqslant 1 / 2^{k}\right. \text { and } \\
&\left.d(0, x a) \geqslant 1 / 2^{k} \text { and } a \cdot B_{k} \subset \bar{B}_{n} \text { and } B_{k} \cdot a \subset \bar{B}_{n}\right\},
\end{aligned}
$$

where

$$
\begin{aligned}
& B_{k}=\left\{x \in X: d(0, x)<1 / 2^{k}\right\}, \\
& \bar{B}_{n}=\left\{x \in X: d(0, x) \leqslant 1 / 2^{n}\right\} ;
\end{aligned}
$$

put

$$
F_{n}^{k}=\left\{a \in W: a^{-1} \cdot B_{k} \subset \bar{B}_{n}, B_{k} \cdot a^{-1} \subset \bar{B}_{n}, a \cdot B_{k} \subset \bar{B}_{n}, B_{k} \cdot a \subset \bar{B}_{n}\right\} .
$$

It is easy to check that $F_{n}^{k}=W \cap E_{n}^{k}$. Each $E_{n}^{k}$ is closed because multiplication is continuous. The family $\left(F_{n}^{k}: k, n \in \mathbf{N}\right)$ is a regular system for $W$ : conditions (i)-(iii) have straightforward verifications. Let us prove condition (iv).

Let $\left(k_{n}\right)$ be a sequence of natural numbers. We remark that $\left(a^{-1}: a \in \bigcap_{n} F_{n}^{k_{n}}\right)$ is an equicontinuous family - both on the right and on the left-of linear operators on $X$.

Thus, if $\left(a_{s}\right)$ is a Cauchy sequence in $\bigcap_{n} F_{n}^{k_{n}}$, the formula

$$
a_{s^{\prime}}^{-1}-a_{s^{\prime \prime}}^{-1}=a_{s^{\prime \prime}}^{-1}\left(a_{s^{\prime \prime}}-a_{s^{\prime}}\right) a_{s^{\prime}}^{-1}
$$

shows that $\left(a_{s}^{-1}\right)$ is also a Cauchy sequence. Let $a_{0}$ and $b_{0}$ be the limits in $X$ of $\left(a_{s}\right)$ and $\left(a_{s}^{-1}\right)$ respectively. Then, by the continuity of multiplication, $a_{0} b_{0}=b_{0} a_{0}=e$ and $a_{0} \in \bigcap_{n} F_{n}^{k_{n}}$. This proves that $\bigcap_{n} F_{n}^{k_{n}}$ is closed in $X$ and $\left(F_{n}^{k}: n, k \in \mathbf{N}\right)$ is a regular system for $W$. By Theorem $11, W$ is an $F_{\text {os }}$ in $X$.

Let us now put $f(x)=x^{-1}$ for $x$ in $W$. It is clear that $f\left(F_{n}^{k}\right)=F_{n}^{k}$ for each $k$ and $n$ in $N$. The formulae $a-b=b\left(b^{-1}-a^{-1}\right) a$ and $a^{-1}-b^{-1}=b^{-1}(b-a) a^{-1}$ $\left(a, b \in W\right.$ ) show that for each sequence $\left(\sigma_{n}\right)=\sigma$ the mapping $f \mid \cap_{n} F_{n}^{\sigma_{n}}: \bigcap_{n} F_{n}^{\sigma_{n}} \rightarrow$ $\bigcap_{n} F_{n}^{\sigma_{n}}$ is a uniform isomorphism. Let $F$ be closed in $W$; then $\left(F \cap F_{n}^{k}: k, n \in N\right)$ is a regular system for $F$. Moreover $f\left(F \cap \bigcap_{n} F_{n}^{\sigma_{n}}\right)=f(F) \cap \bigcap_{n} F_{n}^{\sigma_{n}}$ is closed in $X$ as $F \cap \bigcap_{n} F_{n}^{\sigma_{n}}$ is complete.

Therefore the mapping $B: \mathbf{N}^{N} \rightarrow X$ defined by $B(\sigma)=f\left(F \cap \cap_{n} F_{n}^{\sigma_{n}}\right)$ satisfies the hypotheses of Lemma 12.

Since $f(F)=\Pi_{X}(\mathrm{Gr} B)$ and $\Pi_{\left.X\right|_{\mathrm{Gr} B}}$ is open to $F_{\sigma}$ on $\mathrm{Gr} B$, it follows that $f(F)$ is an $F_{\sigma \delta}$ in $X$ and that $f$, being its own inverse, is a Borel function of the second class.

\section{REFERENCES}

1. B. Aupetit, Propriétés spectrales des algèbres de Banach, Lecture Notes in Math., vol. 735, Springer-Verlag, Berlin and New York, 1979.

2. E. Beckenstein, L. Narici, and C. Suffel, Topological algebras, Mathematics Studies, Vol. 24, North-Holland, Amsterdam, 1977.

3. S. Banach, Remarques sur les groupes et corps métriques, Studia Math. 10 (1948), 178-181.

4. V. Klee, Invariant metrics in groups (solution of a problem of Banach), Proc. Amer. Math. Soc. 3 (1952), 484-487.

5. S. Levi and A. Maitra, Borel measurable images of Polish spaces, Proc. Amer. Math. Soc. 92 (1984), 98-102. 
6. A. L. Shields, The spectrum of an operator on an F-space, Proc. Roy. Irish Acad. Sect. A 74 (1974), 291-292.

7. Z. Slodkowski, Borel sets and the spectrum of an operator on an F-space, Proc. Roy. Soc. Edinburgh Sect. A (1981), 257-261.

8. I. A. Vainstein, On closed mappings, Dokl. Akad. Nauk SSSR 57 (1947), 319-323 (Russian).

Dipartimento di Matematica, Universita di Pisa, Via Buonarroti 2, 56100 Pisa, Italy (Current address of S. Levi)

Department of Mathematics, University of California, los Angeles, California 90024

Current address (Z. Slodkowski): Department of Mathematics, University of Illinois, Chicago, Illinois 60680 